\title{
A NOTE ON GRIFFITHS' CONJECTURE ABOUT THE POSITIVITY OF CHERN-WEIL FORMS
}

\author{
FILIPPO FAGIOLI
}

\begin{abstract}
Let $(E, h)$ be a Griffiths semipositive Hermitian holomorphic vector bundle of rank 3 over a complex manifold. In this paper, we prove the positivity of the characteristic differential form $c_{1}(E, h) \wedge$ $c_{2}(E, h)-c_{3}(E, h)$, thus providing a new evidence towards a conjecture by Griffiths about the positivity of the Schur polynomials in the Chern forms of Griffiths semipositive vector bundles. As a consequence, we establish a new chain of inequalities between Chern forms. Moreover, we point out how to obtain the positivity of the second Chern form $c_{2}(E, h)$ in any rank, starting from the well-known positivity of such form if $(E, h)$ is just Griffiths positive of rank 2. The final part of the paper gives an overview on the state of the art of Griffiths' conjecture, collecting several remarks and open questions.
\end{abstract}

\section{INTRODUCTION}

Let $X$ be a complex manifold of dimension $n$ and let $(E, h)$ be a Hermitian holomorphic vector bundle of rank $r$ over $X$. Starting from the Chern curvature tensor $\Theta(E, h) \in \mathcal{A}^{1,1}(X, \operatorname{End}(E))$, let us consider the corresponding Chern forms on $X$ defined, for $0 \leq k \leq r$, as

$$
c_{k}(E, h)=\operatorname{tr}_{\operatorname{End}\left(\Lambda^{k} E\right)}\left(\Lambda^{k} \frac{i}{2 \pi} \Theta(E, h)\right) .
$$

By the Chern-Weil theory the form $c_{k}(E, h)$ represents the Chern class $c_{k}(E)$ of the vector bundle $E$.

Recall that $(E, h) \rightarrow X$ is called Griffiths semipositive (resp. Griffiths positive) if for every $x \in X, v \in E_{x}, \tau \in T_{X, x}$ we have

$$
\left\langle\Theta(E, h)_{x} \cdot v, v\right\rangle_{h}(\tau, \bar{\tau}) \geq 0
$$

(resp. $>0$ and $=0$ if and only if $v$ or $\tau$ is the zero vector), and that a holomorphic vector bundle $\mathcal{E} \rightarrow X$ is called ample if the tautological line bundle $\mathcal{O}_{\mathbb{P}\left(\mathcal{E}^{\vee}\right)}(1) \rightarrow \mathbb{P}\left(\mathcal{E}^{\vee}\right)$ is ample. The notation $\mathbb{P}\left(\mathcal{E}^{\vee}\right)$ stands for the projective bundle of hyperplanes in $\mathcal{E}$. It is well known that a Griffiths positive vector bundle over a compact complex manifold is ample (the converse is not known in general, but it is a conjecture) and that a globally generated vector bundle (i.e, a bundle whose fibers are spanned by the global holomorphic sections) can be equipped with a Hermitian metric which makes it Griffiths semipositive.

Date: January 1, 2022.

2010 Mathematics Subject Classification. Primary: 53C55; Secondary: 57R20, 57R22, 14M15.

Key words and phrases. Chern-Weil forms, Griffiths' conjecture, flag bundles, pushforward formulæ for flag bundles, Schur forms. 
It is natural to expect that certain conditions of positivity on the vector bundle (such as ampleness or Griffiths positivity) impose, in turn, the positivity of objects that derive from it. This kind of positivity issues can be placed at both algebraic and differential geometric level. In both ways, they were considered by Griffiths in [Gri69], where it was raised the question of determining the characteristic classes/forms built out of an ample/Griffiths positive vector bundle that are positive in the appropriate sense. We refer to [Li21, Xia21, Fin20, DF20] as the most recent works offering a detailed explanation of the results regarding Griffiths' program over the years.

At the algebraic level, one of the main results is the celebrated FultonLazarsfeld theorem [FL83], which characterizes the positive polynomials for ample vector bundles of rank $r$ as those belonging to the positive convex cone spanned by the Schur polynomials (see Section 1.2 for a definition).

Taking a vector bundle $(E, h) \rightarrow X$ as before, we can formally evaluate the Schur polynomials on the Chern forms of $(E, h)$, obtaining a family of characteristic differential forms on $X$. These are the so-called Schur forms of $(E, h)$. The Schur forms are special representatives of the Schur classes of $E$, which are the cohomology classes of $X$ obtained by formally evaluating the Schur polynomials in $c_{1}(E), \ldots, c_{r}(E)$. Significant examples are the Chern classes themselves, classes of the form $c_{1}(E) c_{k-1}(E)-c_{k}(E)$ for $2 \leq k \leq r$, and the signed Segre classes. Recall that the total Segre class $1+s_{1}(E)+\cdots+s_{n}(E)$ is the inverse of the total Chern class in $H^{\bullet}(X)$.

The pointwise Hermitianized version of the above mentioned FultonLazarsfeld theorem is known in the literature as Griffiths' conjecture, which states that the positive convex cone spanned by Schur forms of a Griffiths positive vector bundle consists of positive differential forms (see Section 1.1 for definitions of the three main notions of positivity for differential forms).

In this context, it is straightforward to see that (any power of) the first Chern form is strongly positive for Griffiths semipositive vector bundles. Except for $c_{1}(E, h)$, very little is known about the positivity of the other Schur forms. Griffiths proved in Gri69, Appendix to §5.(b)] that the second Chern form of a rank 2 Griffiths positive vector bundle is positive. However, see [Gri69, p. 247], Griffiths himself deemed difficult to adapt his proof to the general case.

In the last few years there has been a renewed interest around Griffiths' conjecture, as important partial results have appeared in the literature. Guler proved in Gul12, Theorem 1.1] the positivity of the signed Segre forms, which are the Schur forms representing the above mentioned signed Segre classes. More recently, it has been shown in [DF20, Main Application] the strong positivity of an entire sub-cone (which includes, for instance, all the signed Segre forms) of the Schur cone. Such sub-cone is spanned by all the possible push-forwards of powers of the Chern curvatures of certain natural line bundles. These are constructed on the (possibly incomplete) flag bundles associated with $(E, h)$. For definitions and basic notions on push-forwards of differential forms see [Dem12, §I].

Strengthening the hypotheses with stronger notions of positivity, such as (dual) Nakano semipositivity (for instance, see [Fin20] for the definitions), the conjecture is proven in [Li21, Proposition 3.1] and in [Fin20, 
Theorem 1.1]. It is also worth mentioning that Griffiths himself proved his own conjecture in [Gri69, Proof of Theorem D] for globally generated vector bundles. However, the problem still remains open in the general case. In Section 3.1 we provide an overview on the state of the art of Griffiths' conjecture, focusing, in particular, on the recent progress. Primarily, the purpose of such overview is to clarify which types of positivity (among those in Definition 1.2) have been obtained for the Schur forms of positive vector bundles. Indeed, the terminology used in the literature is currently not always standard, and sometimes may lead to some confusion.

In this paper, after recalling the basics on positivity of forms and some needed results on push-forwards from flag bundles, we show how to obtain the positivity of the second Chern form $c_{2}(E, h)$ in any rank, if $(E, h)$ is Griffiths positive. This can be easily obtained by adapting the above mentioned Griffiths' result on the positivity of $c_{2}(E, h)$ in rank 2 . It seems that this was first observed by Guler in Gul06, but just as a statement without proof. Even if the argument is simple, we prefer to give full details for the sake of completeness. Furthermore, we extend such result to the semipositive context in Theorem 2.2 below.

Subsequently, we focus on the Griffiths' conjecture in rank 3, for which we prove the positivity of the Schur form

$$
c_{1}(E, h) \wedge c_{2}(E, h)-c_{3}(E, h) .
$$

The starting point to obtain our result is to consider [DF20, Main Theorem] in order to get the form (因) as a push-forward from the complete flag bundle of $E$. But, at the best of our knowledge, we are not able to deduce the positivity of this form instantly from that push-forward.

Hence, the idea presented in this paper is to factorize such push-forward through the projective bundle $\mathbb{P}(E)$ of lines in $E$. Such idea comes from an alternative proof of the positivity of $c_{2}(E, h)$ in rank 3 , given by the author in an earlier version of this paper. Coupling this factorization to an explicit push-forward formula for differential forms, which we establish in Formula (6) below, we find that (因) can be obtained also by pushing forward a positive form on $\mathbb{P}(E)$, leading us to state our main result.

Theorem 0.1. Let $(E, h) \rightarrow X$ be a Griffiths semipositive Hermitian holomorphic vector bundle of rank 3 over a complex manifold. Then the Schur form $c_{1}(E, h) \wedge c_{2}(E, h)-c_{3}(E, h)$ is a positive $(3,3)$-form on $X$.

Consequently, we find that the following chain of inequalities

$$
c_{1}(E, h)^{\wedge 3} \geq c_{1}(E, h) \wedge c_{2}(E, h) \geq c_{3}(E, h)
$$

holds for Griffiths semipositive vector bundles of rank 3 over complex manifolds. Similar inequalities have appeared, for instance, in [Li21, Theorem 3.2] if $h$ is dual Nakano semipositive, and they give a metric counterpart of some well-known inequalities between Chern numbers valid for nef vector bundles on compact Kähler manifolds (see, for instance, [FL83, DPS94, LZ20]). In the setting of Theorem 0.1 if $X$ is compact (not necessarily Kähler) of

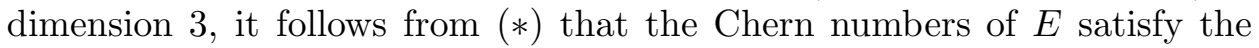
relation

$$
c_{1}^{3} \geq c_{1} c_{2} \geq c_{3} .
$$


Lastly, in Section 3 we collect some concluding remarks and open questions which may serve for further developments of this topic.

Acknowledgements. The author would like to thank his advisor, Simone Diverio, for the help provided during this work, and for the valuable comments on the topics of this paper.

The author is member of GNSAGA (Gruppo Nazionale per le Strutture Algebriche, Geometriche e le loro Applicazioni) of the Istituto Nazionale di Alta Matematica (INdAM) and is partially supported by "Progetti per Avvio alla Ricerca 2020", Project ID: AR120172A92A1FE7, granted by Sapienza Università di Roma.

\section{Preliminaries and notation}

This section is dedicated to introducing the terminology we use throughout this paper. Furthermore, we recall the tools (essentially taken from [DP17] and [DF20]) serving to state Formula (6): a push-forward formula for differential forms on (possibly incomplete) flag bundles. Such formula is particularly useful in the proof of Theorem 0.1 .

1.1. Positivity notions for differential forms. We recall here some basic notions about the positivity of differential forms. The following exposition is taken, mainly, from [HK74] and [Dem12, §III].

Let $V$ be a complex vector space of dimension $n$ and let $\left(e_{1}, \ldots, e_{n}\right)$ be a basis of $V$. The notation $\left(e_{1}^{\vee}, \ldots, e_{n}^{\vee}\right)$ stands for the corresponding dual basis of $V^{\vee}$. For $0 \leq p, q \leq n$, denote by $\Lambda^{p, q} V^{\vee}$ the space of exterior forms of bi-degree $(p, q)$ on $V$. A form $u \in \Lambda^{p, q} V^{\vee}$ is real if $\bar{u}=u$; since $\bar{u} \in \Lambda^{q, p} V^{\vee}$, it follows that $p=q$ in this case. Let $\Lambda_{\mathbb{R}}^{p, p} V^{\vee}$ denote the space of real $(p, p)$-forms.

Definition 1.1. A form $\nu \in \Lambda^{n, n} V^{\vee}$ is called a positive volume form if $\nu=\tau i e_{1}^{\vee} \wedge \bar{e}_{1}^{\vee} \wedge \cdots \wedge i e_{n}^{\vee} \wedge \bar{e}_{n}^{\vee}$ for some $\tau \in \mathbb{R}, \tau \geq 0$.

Of course, this notion is independent on the choice of the basis $\left(e_{1}, \ldots, e_{n}\right)$.

From now on, for $0 \leq p \leq n$ set $q=n-p$. Recall that a $(q, 0)$-form which can be expressed as $\beta_{1} \wedge \cdots \wedge \beta_{q}$ for $\beta_{1}, \ldots, \beta_{q} \in V^{\vee}$ is called decomposable.

Definition 1.2. An exterior form $u \in \Lambda_{\mathbb{R}}^{p, p} V^{\vee}$ is called

- positive, if for every $\beta \in \Lambda^{q, 0} V^{\vee}$ decomposable, $u \wedge i^{q^{2}} \beta \wedge \bar{\beta}$ is a positive volume form;

- Hermitian positive, if for every $\beta \in \Lambda^{q, 0} V^{\vee}, u \wedge i^{q^{2}} \beta \wedge \bar{\beta}$ is a positive volume form;

- strongly positive, if there are decomposable forms $\alpha_{1}, \ldots, \alpha_{N} \in \Lambda^{p, 0} V^{\vee}$ such that $u$ can be expressed as $\sum_{s=1}^{N} i^{p^{2}} \alpha_{s} \wedge \bar{\alpha}_{s}$.

Remark 1.3. The terminology used for the positivity of forms is, currently, not always standard in the literature. We have chosen here to follow the terminology of [Dem12, §III], which is a standard reference for positivity of forms. Note however that [Dem12, §III] does not deal with the intermediate notion of positivity (which is called simply positivity in HK74]). The reason why we call it Hermitian is due to the fact that $u \in \Lambda_{\mathbb{R}}^{p, p} V^{\vee}$ is Hermitian 
positive if and only if for each $\beta, \eta \in \Lambda^{q, 0} V^{\vee},(\beta, \eta) \mapsto u \wedge i^{q^{2}} \beta \wedge \bar{\eta}$ gives a positive semidefinite Hermitian form on $\Lambda^{q, 0} V^{\vee}$.

Remark 1.4. Of course, with an appropriate modification of Definition 1.2, one can define the strict notions of positivity for forms. Mainly, we are dealing here with Griffiths semipositive vector bundles, so it is more natural to consider the three notions given in Definition [1.2, rather than the strict ones. Moreover, note that some authors (see, for instance, Zhe00, Gul12, Li21]) use the term non-negative, or semi-positive, (resp. positive) instead of positive (resp. strictly positive).

Example 1.5. Let $\xi \in \Lambda^{p, 0} V^{\vee}$, then $i^{p^{2}} \xi \wedge \bar{\xi}$ is a Hermitian positive form. Indeed, for every $\beta \in \Lambda^{q, 0} V^{\vee}$, the wedge product $\xi \wedge \beta$ equals $\lambda e_{1}^{\vee} \wedge \cdots \wedge e_{n}^{\vee}$

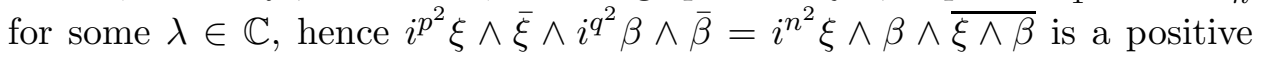
volume form.

Let $\mathrm{WP}^{p} V^{\vee}, \mathrm{HP}^{p} V^{\vee}$ and $\mathrm{SP}^{p} V^{\vee}$ denote respectively the closed positive convex cones contained in $\Lambda_{\mathbb{R}}^{p, p} V^{\vee}$ spanned by positive, Hermitian positive and strongly positive forms. The notations WP and SP are taken from [HK74. It is straightforward to see that, in general,

$$
\mathrm{SP}^{p} V^{\vee} \subseteq \mathrm{HP}^{p} V^{\vee} \subseteq \mathrm{WP}^{p} V^{\vee}
$$

Remark 1.6. The two inclusions in Formula (1) become equalities when $p=0,1, n-1, n$. Indeed, if $p=0, n$ all the positivity notions in Definition 1.2 do coincide. If $p=1$, the one-to-one correspondence between Hermitian forms and real $(1,1)$-forms shows by a diagonalization argument that every positive $(1,1)$-form is strongly positive. By duality, it is also true if $p=n-1$.

If $\mathrm{K}$ is a convex cone in $\Lambda_{\mathbb{R}}^{q, q} V^{\vee}$, then its dual cone is

$$
\mathrm{K}^{*}:=\left\{u \in \Lambda_{\mathbb{R}}^{p, p} V^{\vee} \mid u \wedge v \text { is a positive volume form } \forall v \in \mathrm{K}\right\} .
$$

By Definition 1.2. $\mathrm{WP}^{p} V^{\vee}=\left(\mathrm{SP}^{q} V^{\vee}\right)^{*}$ and given that the bidual of a convex cone is equal to its closure, we have that $\mathrm{SP}^{p} V^{\vee}=\left(\mathrm{WP}^{q} V^{\vee}\right)^{*}$.

We need the following characterization of Hermitian positivity, which follows from the more general [HK74, Theorem 1.2].

Proposition 1.7. A form $u \in \Lambda_{\mathbb{R}}^{p, p} V^{\vee}$ is Hermitian positive if and only if there are $\xi_{1}, \ldots, \xi_{N} \in \Lambda^{p, 0} V^{\vee}$ such that $u=\sum_{s=1}^{N} i^{p^{2}} \xi_{s} \wedge \bar{\xi}_{s}$.

From Proposition 1.7, we deduce that the wedge product of two Hermitian positive forms is again Hermitian positive (of course, an analogous property holds for strongly positive forms by definition). Moreover, it is now clear that the dual cone $\left(\mathrm{HP}^{q} V^{\vee}\right)^{*}$ equals $\mathrm{HP}^{p} V^{\vee}$.

We also want to recall that, sometimes, Proposition 1.7 is given in the literature as a definition; see for instance [Gri69, p. 240] and [Li21, §2].

Remark 1.8. If $2 \leq p \leq n-2$, the two inclusions in Formula (11) are strict. To see this, it is sufficient to observe that the Hermitian positive form $i^{p^{2}} \xi \wedge \bar{\xi}$ is strongly positive if and only if $\xi$ is decomposable (see [Dem12, §III, (1.10) Remark] and [HK74, Proposition 1.5]). Given that, for instance, the $(p, 0)$ form $\left(e_{1}^{\vee} \wedge e_{2}^{\vee}+e_{3}^{\vee} \wedge e_{4}^{\vee}\right) \wedge e_{5}^{\vee} \wedge \cdots \wedge e_{p+2}^{\vee}$ is not decomposable, we deduce that $\mathrm{SP}^{p} V^{\vee} \subsetneq \mathrm{HP}^{p} V^{\vee}$. Since the duality of cones reverses the inclusions, we get also that $\mathrm{HP}^{p} V^{\vee} \subsetneq \mathrm{WP}^{p} V^{\vee}$. 
An explicit example of a positive form which is not Hermitian positive can be found in [HK74, p. 50]. As a byproduct, [HK74] constructs a positive form and a Hermitian positive form, for which their wedge product is a negative volume form. Therefore, unlike $\mathrm{SP}^{p} V^{\vee}$ and $\mathrm{HP}^{p} V^{\vee}$, the cone of positive forms is not stable under wedge product (compare this with [BP13]).

Finally, it is useful to recall the following characterization of positivity.

Proposition 1.9. A form $u \in \Lambda_{\mathbb{R}}^{p, p} V^{\vee}$ is positive if and only if, equivalently,

(1) for every vector subspace $S \subseteq V$ with $\operatorname{dim}_{\mathbb{C}} S=p$, the restriction $\left.u\right|_{S}$ is a positive volume form on $S$;

(2) for every $w_{1}, \ldots, w_{p} \in V,(-i)^{p^{2}} u\left(w_{1}, \ldots, w_{p}, \bar{w}_{1}, \ldots, \bar{w}_{p}\right) \geq 0$.

Of course, all of the definitions and results given in this section can be extended to a complex manifold $X$. It is sufficient to take $V=T_{X, x}$ for every $x \in X$ and to check that all the concepts expressed here are independent by change of holomorphic coordinates. This follows from Definition 1.1.

If $E \rightarrow X$ is a complex vector bundle and $0 \leq p, q \leq n$, then $\mathcal{A}^{p, q}(X, E)$ stands for the space $C^{\infty}\left(X, \Lambda^{p, q} T_{X}^{\vee} \otimes E\right)$ of differential $(p, q)$-forms on $X$ with values in $E$. In particular, $\mathcal{A}^{p, q}(X)$ denotes the space of differential $(p, q)$-forms on $X$. Similarly, we use the notations $\mathcal{A}_{\mathbb{R}}^{p, q}(X)$ and $\mathcal{A}^{k}(X)$.

1.2. Generalized Schur classes/forms. Fix $k \in \mathbb{N}$ and denote by $\Lambda(k, r)$ the set of partitions of $k$ in $r$ parts, i.e., the set of all $\sigma=\left(\sigma_{1}, \ldots, \sigma_{k}\right) \in \mathbb{N}^{k}$ such that $r \geq \sigma_{1} \geq \cdots \geq \sigma_{k} \geq 0$ and $|\sigma|=\sum_{j=1}^{k} \sigma_{j}=k$. To $\sigma \in \Lambda(k, r)$ we associate the Schur polynomial $S_{\sigma} \in \mathbb{Z}\left[c_{1}, \ldots, c_{r}\right]$ of weighted degree $2 k$ (we regard $c_{j}$ as having degree $2 j$ ), which is defined as

$$
S_{\sigma}\left(c_{1}, \ldots, c_{r}\right):=\operatorname{det}\left(c_{\sigma_{i}+j-i}\right)_{1 \leq i, j \leq k} .
$$

By convention, $c_{0}=1$ and $c_{\ell}=0$ if $\ell<0$ or $\ell>r$.

As $\sigma \in \Lambda(k, r)$ varies, the Schur polynomials form a basis for the $\mathbb{Q}$-vector space of degree $2 k$ weighted homogeneous polynomials in $r$ variables, and the product of two Schur polynomials is a positive linear combination of Schur polynomials. Hence, Schur polynomials generate a positive convex cone closed under product.

From now on, let $X$ be a complex manifold of dimension $n$ and let $(E, h)$ be a Hermitian holomorphic vector bundle of rank $r$ over $X$. The definitions of Schur forms (resp. classes) of $(E, h)$ (resp. E) are already given in the Introduction. We denote by $S_{\sigma}(E, h)$ the Schur form associated to the partition $\sigma$. Similarly, $S_{\sigma}(E)$ is the Schur class associated to $\sigma$. Clearly, $S_{\sigma}(E, h)$ represents the cohomology class $S_{\sigma}(E)$. For instance,

$$
S_{\sigma}(E, h)= \begin{cases}c_{k}(E, h) & \text { if } \sigma=(k, 0, \ldots, 0), \\ c_{1}(E, h) \wedge c_{k-1}(E, h)-c_{k}(E, h) & \text { if } \sigma=(k-1,1,0, \ldots, 0), \\ (-1)^{k} s_{k}(E, h) & \text { if } \sigma=(1, \ldots, 1),\end{cases}
$$

where, in all the three cases above, $\sigma \in \Lambda(k, r)$, and $s_{k}(E, h)$ stands for the $k$-th Segre form of $(E, h)$, which represents the Segre class $s_{k}(E)$.

Now, we want to introduce a larger family of cohomology classes (resp. differential forms) which are given by dropping the assumption that $\sigma$ is a partition in $\Lambda(k, r)$. The following notation is taken from [DP17, §4], and will be essential in order to state Formula (6). 
Definition 1.10. Let $\sigma=\left(\sigma_{1}, \ldots, \sigma_{k}\right) \in \mathbb{Z}^{k}$ be a sequence of integers. We define a cohomology class

$$
s_{\sigma}(E):=\operatorname{det}\left(s_{\sigma_{i}+j-i}(E)\right)_{1 \leq i, j \leq k}
$$

in $H^{2|\sigma|}(X, \mathbb{Z})$, where, as usual, $s_{0}(E)=1$ and $s_{\ell}(E)=0$ if $\ell \notin[0, n]$.

The relationship between Schur classes and Definition 1.10 is given in the following.

Example 1.11. Let $\sigma$ be a partition in $\Lambda(k, r)$. It follows from the well-known Jacobi-Trudi identities that

$$
s_{\sigma}(E)=(-1)^{|\sigma|} S_{\sigma^{\prime}}(E),
$$

where $\sigma^{\prime}$ is the conjugate partition of $\sigma$, obtained through the transposition of the Young diagram of $\sigma$. For instance, by Formula (2) the partition $(1, \ldots, 1)$ gives $(-1)^{k} c_{k}(E)$, which is the $k$-th signed Chern class, while the Segre class $s_{k}(E)$ is associated to the partition $(k, 0, \ldots, 0)$.

Note that the sign $(-1)^{|\sigma|}$ in Formula (21) is due to the fact that the definition of Schur classes involves Chern classes, while Definition 1.10 is given in terms of Segre classes. The reason why we choose the Segre classes' approach in Definition 1.10 is to make our notation uniform with that of [DP17].

Similarly to Definition 1.10, we can associate to any $\sigma \in \mathbb{Z}^{k}$ the differential form $s_{\sigma}(E, h):=\operatorname{det}\left(s_{\sigma_{i}+j-i}(E, h)\right)$. Inspired by Formula (2), we call $s_{\sigma}(E, h)$ (resp. $\left.s_{\sigma}(E)\right)$ the generalized Schur form (resp. class) associated to $\sigma$.

1.3. Flag bundles. Now we introduce some notation about flag bundles associated to the holomorphic vector bundle $E \rightarrow X$. Fix a sequence of integers $\rho=\left(\rho_{0}, \ldots, \rho_{m}\right)$ of the form $0=\rho_{0}<\rho_{1}<\ldots<\rho_{m-1}<\rho_{m}=r$, the flag bundle of $E$ of type $\rho$ is the holomorphic fiber bundle

$$
\pi_{\rho}: \mathbb{F}_{\rho}(E) \rightarrow X
$$

where the fiber over $x \in X$ is the flag manifold $\mathbb{F}_{\rho}\left(E_{x}\right)$, whose points are flags of the form $\left\{0_{x}\right\}=V_{x, \rho_{0}} \subset \cdots \subset V_{x, \rho_{j}} \subset \cdots \subset V_{x, \rho_{m}}=E_{x}$ with $\operatorname{dim}_{\mathbb{C}} V_{x, \rho_{j}}=\rho_{j}$. Over $\mathbb{F}_{\rho}(E)$ we have a tautological flag

$$
U_{\rho, 0} \subset \cdots \subset U_{\rho, j} \subset \cdots \subset U_{\rho, m}
$$

of vector sub-bundles of $\pi_{\rho}^{*} E$, where, for every $0 \leq j \leq m$, the fiber of $U_{\rho, j}$ over the point $\left\{0_{x}\right\} \subset \cdots \subset V_{x, \rho_{j}} \subset \cdots \subset E_{x}$ is $V_{x, \rho_{j}}$, hence, the rank of $U_{\rho, j}$ is $\rho_{j}$. We note, in particular, that $U_{\rho, 0}=(0)$ and $U_{\rho, m}=\pi_{\rho}^{*} E$.

Example 1.12. If $\rho$ is the sequence $(0,1, r)$ then $\mathbb{F}_{\rho}(E)$ equals the projective bundle of lines in $E$, which we denote by $\mathbb{P}(E)$. In this case, the tautological filtration (3) consists of one proper sub-bundle only, namely $U_{(0,1, r), 1}$, which equals, by definition, the tautological line bundle $\mathcal{O}_{\mathbb{P}(E)}(-1)$.

In the particular case of complete flag bundles, i.e. $m=r$, we shall drop the subscript $\rho$ and simply write $\pi, \mathbb{F}(E)$ and $U_{j}$.

We denote by $\pi_{\rho}^{r}: \mathbb{F}(E) \rightarrow \mathbb{F}_{\rho}(E)$ the obvious projection which sends the complete flag $\left\{0_{x}\right\} \subset V_{x, 1} \subset \cdots \subset V_{x, r-1} \subset E_{x}$ to the (partial) flag $\left\{0_{x}\right\} \subset V_{x, \rho_{1}} \subset \cdots \subset V_{x, \rho_{m-1}} \subset E_{x}$ of $\mathbb{F}_{\rho}(E)$. 
Finally, note that the natural commutative diagram

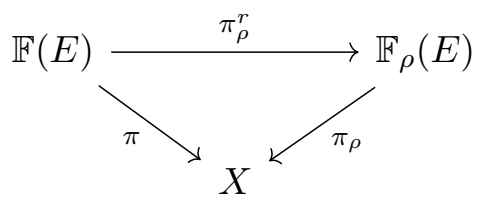

of projections between flag bundles induces, for $0 \leq k \leq n$, a commutative diagram of push-forwards (intended as integration along the fibers) at the level of differential forms (see [Dem12, §I, (2.14) Theorem])

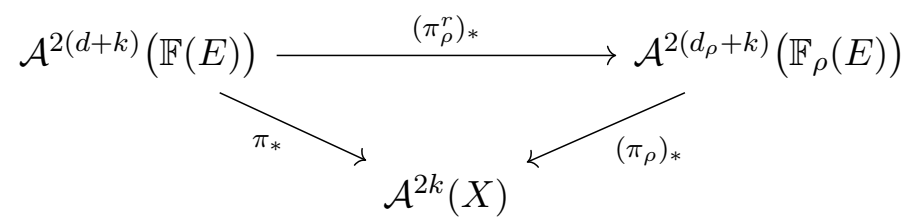

where $d$ (resp. $d_{\rho}$ ) stands for the relative dimension of $\pi$ (resp. $\pi_{\rho}$ ). We also use the same notation, namely $\pi_{*}$ and $\left(\pi_{\rho}\right)_{*}$, for the push-forwards induced in cohomology by integration along the fibers.

1.4. Push-forward formulæ. Following again the notation of [DP17], denote by $\xi_{1}, \ldots, \xi_{r}$ the (virtual) Chern roots of $\pi_{\rho}^{*} E^{\vee}$. Let $P$ be a polynomial with the appropriate symmetries which ensure that $P\left(\xi_{1}, \ldots, \xi_{r}\right) \in$ $H^{\bullet}\left(\mathbb{F}_{\rho}(E)\right)$. In this setting, we now recall the determinantal version of Darondeau-Pragacz formula [DP17, Proposition 4.2], given with a little modification as follows.

Proposition 1.13. Take the polynomial $P$ as before, and write the class $P\left(\xi_{1}, \ldots, \xi_{r}\right)$ as $\sum a_{\lambda} \xi_{1}^{\lambda_{1}} \cdots \xi_{r}^{\lambda_{r}}$. If $\nu$ is the increasing sequence of integers determined by $\rho$ as:

$$
\nu_{i}=r-\rho_{s} \quad \text { for } r-\rho_{s}<i \leq r-\rho_{s-1}
$$

(in particular, if $m=r$ the sequence $\nu$ is given by $\nu_{i}=i-1$ ), then, in terms of generalized Schur classes, we have

$$
\left(\pi_{\rho}\right)_{*} P\left(\xi_{1}, \ldots, \xi_{r}\right)=\sum a_{\lambda} s_{(\lambda-\nu) \leftarrow}(E)
$$

where the notation $\left(\sigma_{1}, \ldots, \sigma_{r}\right)^{\leftarrow}$ stands for $\left(\sigma_{r}, \ldots, \sigma_{1}\right)$ and the difference of $\lambda$ and $\nu$ is defined componentwise.

Remark 1.14. Proposition 1.13 is stated a little differently from [DP17, Proposition 4.2]. Indeed, the original statement of Darondeau and Pragacz is given in terms of the Chern roots of $U_{\rho, m-1}^{\vee}$ (which is the greatest proper tautological sub-bundle of $\pi_{\rho}^{*} E^{\vee}$ ), while we need a push-forward formula involving all the Chern roots of $\pi_{\rho}^{*} E^{\vee}$.

However, we have chosen to omit the proof of Proposition 1.13 since it is essentially the same as [DP17, Proposition 4.2]. The only relevant difference is that we use [DP17, Proposition 1.2] in the first step of the proof instead of [DP17, Theorem 1.1].

We now move to the differential forms level and suppose thereafter that $(E, h) \rightarrow X$ is a Hermitian holomorphic vector bundle. 
Consider the filtration (3) of tautological vector bundles over $\mathbb{F}_{\rho}(E)$, all of them equipped with the respective restriction metrics induced by $h$. One can form, for $j=1, \ldots, m$, tautological line bundles

$$
Q_{\rho, j}:=\operatorname{det}\left(U_{\rho, m-j+1} / U_{\rho, m-j}\right)
$$

endowed with the determinant of the quotient metrics. By a slight abuse of notation, denote again by $h$ all these above mentioned metrics, and let

$$
\Xi_{\rho, j}:=\frac{i}{2 \pi} \Theta\left(Q_{\rho, j}, h\right) \in \mathcal{A}_{\mathbb{R}}^{1,1}\left(\mathbb{F}_{\rho}(E)\right)
$$

be the first Chern form $c_{1}\left(Q_{\rho, j}, h\right)$. Of course, $\Xi_{\rho, j}$ represents $c_{1}\left(Q_{\rho, j}\right)$.

When $m=r$, we drop the subscript $\rho$ and simply write $Q_{j}$ and $\Xi_{j}$. Note that, in this case, $\xi_{j}=-c_{1}\left(Q_{j}\right)=-\left[\Xi_{j}\right]$.

Given a homogeneous polynomial $F$ in $m$ variables of degree $d_{\rho}+k$, we now want to compute the push-forward $\left(\pi_{\rho}\right)_{*} F\left(\Xi_{\rho, 1}, \ldots, \Xi_{\rho, m}\right)$ in terms of generalized Schur forms of $(E, h)$. Clearly, at the cohomology level we have that $F\left(c_{1}\left(Q_{\rho, 1}\right), \ldots, c_{1}\left(Q_{\rho, m}\right)\right) \in H^{2\left(d_{\rho}+k\right)}\left(\mathbb{F}_{\rho}(E)\right)$. Therefore, there exists a polynomial in the virtual Chern roots of $\pi_{\rho}^{*} E^{\vee}$ called

$$
f\left(\xi_{1}, \ldots, \xi_{r}\right)=\sum_{|\lambda|=d_{\rho}+k} b_{\lambda} \xi_{1}^{\lambda_{1}} \cdots \xi_{r}^{\lambda_{r}}
$$

such that, for $0 \leq j \leq m$ and $s_{j}:=r-\rho_{m-j}$,

$$
f\left(\xi_{1}, \ldots, \xi_{r}\right)=F\left(\ldots,-\sum_{\ell=s_{j-1}+1}^{s_{j}} \xi_{\ell}, \ldots\right)=F\left(c_{1}\left(Q_{\rho, 1}\right), \ldots, c_{1}\left(Q_{\rho, m}\right)\right),
$$

i.e., the polynomial $f$ has by construction the appropriate symmetries for which $f\left(\xi_{1}, \ldots, \xi_{r}\right)$ can be considered as a cohomology class of $\mathbb{F}_{\rho}(E)$.

Note in particular that, if $m=r$, the Chern roots of $\pi^{*} E^{\vee}$ are not virtual. Hence, the only relevant symmetry in this case is $f=(-1)^{d_{\rho}+k} F$.

Thanks to [DF20, Theorem 3.5] we are able to identify the push-forward of the form $F\left(\Xi_{\rho, 1}, \ldots, \Xi_{\rho, m}\right)$ on the manifold $X$. Accordingly to DP17, Proposition 1.2], the explicit expression of such push-forward is given in [DF20] as the coefficient of certain monomial of a polynomial depending on $n, r$ and $f$, formally evaluated on the Segre forms $s_{j}(E, h)$ 's. Since the explicit expression of the push-forward is obviously independent of the method we use to compute it, we prefer for our purposes to couple [DF20, Theorem 3.5] with Proposition [1.13, obtaining the following identity

$$
\left(\pi_{\rho}\right)_{*} F\left(\Xi_{\rho, 1}, \ldots, \Xi_{\rho, m}\right)=\sum_{|\lambda|=d_{\rho}+k} b_{\lambda} s_{(\lambda-\nu)^{\leftarrow}}(E, h),
$$

where $\nu$ is given as in Proposition 1.13 and the $b_{\lambda}$ 's are the coefficients of $f$.

\section{Proof of the MAin Results}

In what follows, we proceed to observe that the positivity of the second Chern form is implied by the Griffiths semipositivity of the curvature tensor. Such argument is central in the proof Theorem 0.1 .

Let $(E, h)$ be a Griffiths positive Hermitian holomorphic vector bundle of rank 2 over a complex manifold $X$. Thanks to Gri69, Appendix to §5.(b)], 
we know that the second Chern form $c_{2}(E, h)$ is positive. The argument used by Griffiths in the proof consists in the following. First, by Proposition 1.9. one can assume that $X$ is a complex surface. Of course, this assumption is not allowed if one wants to show the Hermitian (or strong) positivity of $c_{2}(E, h)$ (cf. with Remarks 1.6 and 1.8). However, in rank 2 the curvature is a $2 \times 2$ matrix of $(1,1)$-forms, thus

$$
c_{2}(E, h)=-\frac{1}{4 \pi^{2}} \operatorname{det} \Theta(E, h) .
$$

Given that the Hermitian forms associated to the diagonal entries of the matrix $i \Theta(E, h)$ are positive definite, one can perform a simultaneous diagonalization of such Hermitian forms. After the diagonalization, using that $\operatorname{dim} X=2$, to show that $-\operatorname{det} \Theta(E, h)$ is positive it is sufficient to apply the Schwarz inequality coupled with the definition of Griffiths positivity.

Now, suppose that $(E, h)$ is Griffiths semipositive. Once fixed a strictly positive $(1,1)$-form $\omega$ on $X$ and $\varepsilon>0$, we can apply the above argument to the $\operatorname{Herm}(E)$-valued $(1,1)$-form

$$
i \Theta(E, h)+\varepsilon \omega \otimes \operatorname{Id}_{E} .
$$

Passing to the limit for $\varepsilon \rightarrow 0$ we get the following.

Lemma 2.1. Given $(E, h) \rightarrow X$ Griffiths semipositive of rank 2, the differential form $c_{2}(E, h)$ is positive.

2.1. Positivity of $c_{2}$ in any rank. Let $(E, h) \rightarrow X$ be a Hermitian holomorphic vector bundle of rank $r$, and denote by $\Theta_{\alpha \beta}$ the entries of the curvature matrix. In general, we have that

$$
\begin{aligned}
c_{2}(E, h) & =\operatorname{tr}_{\operatorname{End}\left(\Lambda^{2} E\right)}\left(\Lambda^{2} \frac{i}{2 \pi} \Theta(E, h)\right) \\
& =-\frac{1}{4 \pi^{2}} \sum_{1 \leq \alpha<\beta \leq r}\left(\Theta_{\alpha \alpha} \wedge \Theta_{\beta \beta}-\Theta_{\alpha \beta} \wedge \Theta_{\beta \alpha}\right) .
\end{aligned}
$$

If $(E, h)$ is Griffiths positive, every diagonal entry $\Theta_{\alpha \alpha}$ of $\Theta(E, h)$ gives a positive definite Hermitian form. Thanks to a suggestion of an anonymous referee we realized that it is possible to apply Gri69, Appendix to §5.(b)] to all the summands in the last member of Formula (7). This because each summand is by definition the determinant of a $2 \times 2$ principal sub-matrix of the curvature matrix. From this follows the positivity of $c_{2}(E, h)$ in any rank.

In the semipositive case, the positivity of $c_{2}(E, h)$ is obtained by applying Lemma 2.1 (instead of the above argument) to all the summands in the last member of Formula (7). This proves the following.

Theorem 2.2. Let $(E, h)$ be a Griffiths semipositive Hermitian holomorphic vector bundle over a complex manifold. Then the second Chern form $c_{2}(E, h)$ is a positive $(2,2)$-form.

2.2. Positivity of $c_{1} c_{2}-c_{3}$ in rank 3. From now on, suppose that $r=3$. Let $p:=\pi_{(0,1,3)}: \mathbb{P}(E) \rightarrow X$ be the projective bundle of lines in $E$ with associated tautological short exact sequence

$$
0 \rightarrow \mathcal{O}_{\mathbb{P}(E)}(-1) \hookrightarrow p^{*} E \rightarrow Q:=p^{*} E / \mathcal{O}_{\mathbb{P}(E)}(-1) \rightarrow 0
$$


over $\mathbb{P}(E)$. Note that the quotient bundle $Q \rightarrow \mathbb{P}(E)$ is Griffiths semipositive of rank 2 with respect to the natural quotient metric induced by $(E, h)$ (and denoted again by $h$ ). Consequently, the $(1,1)$-form $c_{1}(Q, h)$ is strongly positive and, by Lemma 2.1, $c_{2}(Q, h)$ is positive.

We are now ready to prove the positivity of the Schur form

$$
S_{(2,1,0)}(E, h)=c_{1}(E, h) \wedge c_{2}(E, h)-c_{3}(E, h) .
$$

Remark 2.3. The main idea behind the next proof was developed for the first time by the author in an alternative proof of the positivity of $c_{2}(E, h)$ in rank 3. Since the positivity of $c_{2}(E, h)$ for Griffiths semipositive vector bundles is the content of Theorem 2.2, we omit such an alternative proof to make te exposition more compact.

Proof of Theorem 0.1. Following the notation of Section 1.4, let $\xi_{1}, \xi_{2}, \xi_{3}$ be the Chern roots of $\pi^{*} E^{\vee}$. They are given by the tautological filtration (3) (where $r=m=3$ ), for which $\pi^{*} E$ splits (non canonically) as a differentiable vector bundle over $\mathbb{F}(E)$ as $Q_{1} \oplus Q_{2} \oplus Q_{3}$.

By the commutativity of the following diagram of projections

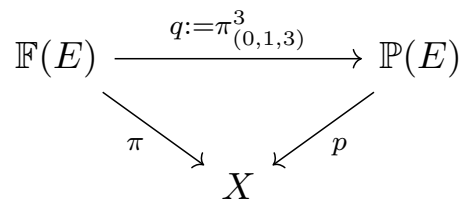

(cf. with the commutative diagram (41) above) we see that $\xi_{1}$ and $\xi_{2}$ are also the Chern roots of $q^{*} Q^{\vee}$, where $Q \rightarrow \mathbb{P}(E)$ is the rank 2 tautological quotient bundle introduced before. Indeed,

$$
q^{*} Q=q^{*}\left(p^{*} E / \mathcal{O}_{\mathbb{P}(E)}(-1)\right)=\pi^{*} E / U_{1}
$$

and, since $\mathbb{F}(E)$ coincides with $\mathbb{F}(Q)=\mathbb{P}(Q)$, we deduce that there is a non canonical isomorphism

$$
q^{*} Q \cong_{C^{\infty}} \pi^{*} E / U_{2} \oplus U_{2} / U_{1}=Q_{1} \oplus Q_{2} .
$$

By Formula (6) applied to $\pi: \mathbb{F}(E) \rightarrow X$ and where $F\left(\Xi_{1}, \Xi_{2}, \Xi_{3}\right)$ is taken to be the monomial $\Xi_{1}^{\wedge 4} \wedge \Xi_{2}^{\wedge 2} \wedge \Xi_{3}^{\wedge 0}$, we have the equality

$$
\pi_{*}\left[\Xi_{1}^{\wedge 4} \wedge \Xi_{2}^{\wedge 2} \wedge \Xi_{3}^{\wedge 0}\right]=s_{(-2,1,4)}(E, h)=S_{(2,1,0)}(E, h) .
$$

Observe however that, a priori, we do not conclude anything about the positivity of $S_{(2,1,0)}(E, h)$ using only Equation (9), since, for instance, the line bundle $Q_{2}$ is not positive in general. Therefore, we apply again Formula (6), but to the flag bundle $q: \mathbb{P}(Q) \rightarrow \mathbb{P}(E)$, getting

$$
q_{*}\left[\Xi_{1}^{\wedge 4} \wedge \Xi_{2}^{\wedge 2}\right]=s_{(1,4)}(Q, h)=c_{1}(Q, h) \wedge c_{2}(Q, h)^{\wedge 2},
$$

where the last equality holds given that $c_{3}(Q, h) \equiv 0$ and $c_{4}(Q, h) \equiv 0$.

Hence, the chain of equalities

$$
\begin{aligned}
& p_{*}\left[c_{1}(Q, h) \wedge c_{2}(Q, h)^{\wedge 2}\right]=p_{*} q_{*}\left[\Xi_{1}^{\wedge 4} \wedge \Xi_{2}^{\wedge 2}\right] \\
& =\pi_{*}\left[\Xi_{1}^{\wedge 4} \wedge \Xi_{2}^{\wedge 2} \wedge \Xi_{3}^{\wedge 0}\right] \text { by commutativity of (8) } \\
& =S_{(2,1,0)}(E, h) \\
& \text { by Formula (9) }
\end{aligned}
$$

follows, by applying $p_{*}$ to both members of Formula (10). 
Since $Q \rightarrow \mathbb{P}(E)$ is Griffiths semipositive, by Lemma 2.1 we know that $c_{2}(Q, h)$ is a positive form on $\mathbb{P}(E)$. Thanks to [BP13, Theorem 1], the square of a positive $(2,2)$-form is also positive. Hence $c_{1}(Q, h) \wedge c_{2}(Q, h)^{\wedge 2}$ is positive, given that it is the wedge product of a strongly positive and of a positive form. Consequently, $S_{(2,1,0)}(E, h)$ must be a positive form on $X$, since it is the push-forward of a positive differential form.

Remark 2.4. For dimension and bi-degree reasons (see Remark 1.6) we underline that if $\operatorname{dim} X \leq 3$, then $c_{2}(E, h)$ is strongly positive, and if $\operatorname{dim} X \leq 4$, then $S_{(2,1,0)}(E, h)$ is strongly positive, too.

From the (strong) positivity of the second Segre form

$$
s_{2}(E, h)=c_{1}(E, h)^{\wedge 2}-c_{2}(E, h)
$$

(see Gul12, DF20]) and from Theorem 0.1 we deduce the following.

Corollary 2.5. If $(E, h) \rightarrow X$ is Griffiths semipositive of rank 3 over a complex manifold, then the following pointwise inequalities hold

$$
c_{1}(E, h)^{\wedge 3} \geq c_{1}(E, h) \wedge c_{2}(E, h) \geq c_{3}(E, h) .
$$

In addition, if $X$ is compact and 3-dimensional, the following chain of inequalities between Chern numbers also holds

$$
\int_{X} c_{1}(E)^{3} \geq \int_{X} c_{1}(E) c_{2}(E) \geq \int_{X} c_{3}(E) .
$$

As already mentioned in the Introduction, the inequalities in (11) can be deduced by [Li21, Theorem 3.2] only if $h$ is dual Nakano semipositive. Thus, Corollary 2.5] is in some sense a generalization of [Li21, Theorem 3.2] in rank 3.

Remark 2.6. It is well-known that a vector bundle admitting a Griffiths semipositive metric is nef (see [DPS94] for a definition). Recall that the Chern numbers of a nef vector bundle on a compact $n$-dimensional Kähler manifold are bounded above by the Chern number $c_{1}^{n}$ ([DPS94, Corollary 2.6]) and below by the Euler number $c_{n}$ ([LZ20, Theorem 2.9]). See also [Li21, Remark 3.3]. Therefore, assuming that $X$ is also Kähler the first and the second inequalities in (12) are a particular case of [DPS94, Corollary 2.6] and [LZ20, Theorem 2.9] respectively. Moreover, observe that the inequalities in (12) follow from [DPS94, Theorem 2.5] if $X$ is Kähler.

Remark 2.7. It seems difficult to apply the strategy in the proof of Theorem 0.1 to prove the positivity of $c_{3}(E, h)$ in rank 3 . Indeed, in order to factor through the quotient bundle, we need to push-forward only monomials of the form $\Xi_{1}^{\wedge \lambda_{1}} \wedge \Xi_{2}^{\wedge \lambda_{2}}$. But, by Formula (6) we have the equality $\pi_{*}\left[\Xi_{1}^{\wedge 3} \wedge \Xi_{2}^{\wedge 2} \wedge \Xi_{3}\right]=c_{3}(E, h)$ that involves $\Xi_{3}$, which is the pull-back through $q$ of the Chern curvature of $\mathcal{O}_{\mathbb{P}(E)}(-1)$.

However, one can try to adapt the same ideas presented in this work to vector bundles of rank higher than 3 . For example, in rank 4 we could use the tautological quotient of the Grassmann bundle $\mathbb{G}_{2}(E)$ in order to get push-forward formulæ for some Schur forms. The problem here is that the monomials we push-forward contain $c_{2}^{\wedge \ell}, \ell>2$. Therefore, as pointed out for instance in [BP13], we do not know a priori if these powers are positive. 


\section{State of the ART, CONCLUding Remarks AND open QUESTiOnS}

As always, let $(E, h)$ be a rank $r$ Hermitian holomorphic vector bundle over a complex manifold $X$.

3.1. A comparison on the positivity of Schur forms. We take the opportunity here to compare the different notions of positivity (see Definition 1.2) of Schur forms that appear in [BC65, Gri69, Gul12, Li21, Fin20, DF20, which are the works mainly related to Theorem 0.1 .

Recall that we do not deal with the strict notions of positivity (see Remark 1.4). For this, the following exposition can be made slightly more precise, although this does not affect the purpose of the comparison.

Positivity in [BC65]. In [BC65, Definition 5.1] a notion of positivity for elements in $\mathcal{A}^{p, p}(X, \operatorname{End}(E))$ is given. In particular, for $p=1$, such notion includes the so-called Bott-Chern nonnegativity (this terminology is due to [Li21], see below), which requires that the curvature of $(E, h)$ can be expressed, locally, as $A \wedge \bar{A}^{t}$, where $A$ is a matrix of (1,0)-forms of appropriate size. Although [BC65] predates Griffiths' conjecture, from [BC65, Lemma 5.3] one can deduce the Hermitian positivity of the top Chern form of a Bott-Chern nonnegative vector bundle.

Positivity in [Gri69]. The positivity notion considered in [Gri69, p. 240] is, by Proposition 1.7. Hermitian positivity. As already mentioned, Griffiths' conjecture first appears in [Gri69, p. 247], where it is conjectured that for a Griffiths positive vector bundle the cone of positive polynomials in the Chern forms (i.e., the Schur cone) consists of Hermitian positive differential forms. The full conjecture is verified in Gri69, p. 246] for globally generated vector bundles; for more details we refer to [Gri69, Proof of Theorem D].

Moreover, by the characterization of Proposition 1.9, Griffiths proves in [Gri69, Appendix to §5.(b)] the positivity, but not the Hermitian positivity, of $c_{2}(E, h)$, for $(E, h)$ Griffiths positive of rank 2 .

Positivity in Gul12]. The main result of [Gul12] states that the signed Segre forms of a Griffiths positive vector bundle are positive. Actually, Gul12, Theorem 1.1] implicitly proves that such forms are strongly positive, even though the strong positivity is not explicitly observed therein.

Positivity in [Li21. The two positivity notions considered by [Li21] are, by Propositions 1.9 and 1.7, positivity and Hermitian positivity, although in [Li21, §2] are called nonnegativity and strong nonnegativity respectively. In the same spirit of [Gri69, Proof of Theorem D], [Li21, Proposition 3.1] extends the above mentioned result on globally generated vector bundles to the larger family of Bott-Chern nonnegative (see [Li21, Definition 2.1]) vector bundles, showing that the Schur forms of these bundles are Hermitian positive.

Positivity in Fin20]. All the three notions of Definition 1.2 are addressed in [Fin20], although with the terminology of [HK74]. The full Griffiths' conjecture is verified in [Fin20, Theorem 1.1], where it is shown the Hermitian positivity of the Schur forms of a Nakano, or dual Nakano, positive vector bundle. 
It is also worth to recall that Fin20 relates, in a very interesting way, Bott-Chern's notion of positivity with the dual Nakano one. More precisely, [Fin20, Theorem 2.14] states that a Hermitian vector bundle $(E, h) \rightarrow X$ is dual Nakano semipositive if and only if for every $x \in X$ there is a vector space $V$ and a matrix of $(1,0)$-forms $A \in T_{X, x}^{\vee} \otimes \operatorname{Hom}\left(V, E_{x}\right)$ such that $\Theta(E, h)_{x}=A \wedge \bar{A}^{t}$.

In order to have a similar characterization for dual Nakano positivity, we observe that it is sufficient to require, in addition, the invertibility of the operator $\tilde{A} \in \operatorname{Hom}\left(T_{X, x} \otimes E_{x}^{\vee}, V^{\vee}\right)$ naturally associated to $A$. Therefore, both [Li21] and [Fin20] obtain the full Griffiths' conjecture for dual Nakano (semi)positive bundles, but with different methods.

Finally, we recall that [Fin20, Theorem 1.3] establishes the equivalence between Griffiths' conjecture and an open question concerning the so-called positive semidefinite linear preservers, see [Fin20, Open problem].

Positivity in [DF20]. As already mentioned in the Introduction, given a Griffiths semipositive vector bundle $(E, h) \rightarrow X$, DF20, Main Application] shows the strong positivity of a family of differential forms in the Schur cone of $(E, h)$. Such family form a sub-cone which, in the notation of Section 1.4, is spanned by all possible wedge products of all possible push-forwards

$$
\left(\pi_{\rho}\right)_{*}\left(a_{1} \Xi_{\rho, 1}+\cdots+a_{m} \Xi_{\rho, m}\right)^{d_{\rho}+k}
$$

as $k \geq 0, \rho=\left(\rho_{0}, \ldots, \rho_{m}\right)$ and the sequence of integers $a_{1} \geq \cdots \geq a_{m} \geq 0$ vary.

Observe that to $\rho=(0, r-1, r)$ corresponds the bundle $\pi_{\rho}: \mathbb{P}\left(E^{\vee}\right) \rightarrow X$ of hyperplanes in $E$. Hence $\Xi_{\rho, 1}=c_{1}\left(\mathcal{O}_{\mathbb{P}\left(E^{\vee}\right)}(1), h\right)$ and for $\left(a_{1}, a_{2}\right)=(1,0)$ Guler's result [Gul12, Theorem 1.1] on the signed Segre forms is recovered.

3.2. Concluding remarks. If we assume (dual) Nakano semipositivity, then the Schur cone consists of Hermitian positive differential forms. Hence, it is natural to ask the following question.

Question 1. Beside those as in Expression (13) (and wedge products of them), are there in the Schur cone other strongly positive differential forms for (dual) Nakano semipositive vector bundles?

Of course, the best possible result would be that all the Schur cone consists of strongly positive differential forms.

Assume now that the vector bundle is Griffiths semipositive. Although it is not explicitly mentioned in Griffiths' conjecture (as stated in the literature), at this point it seems interesting to ask what is the most natural notion of positivity, among those in Definition 1.2, that we can expect to hold for the Schur forms of the vector bundle. Certainly, the conjecture is still open even requiring the weakest notion of positivity, although, as already said, [DF20, Main Application] shows us that the strong positivity naturally appears in this context. In addition, it is in a sense more natural to require the strong, or Hermitian, positivity of the Schur forms. Indeed, as mentioned in Section 1.2, positive polynomials (i.e., those belonging to the 
Schur cone) are stable under product and so do Hermitian and strongly positive forms; while the wedge product of two positive forms is not necessarily positive (see [Dem12, §III, (1.11) Proposition] and [BP13]).

Summing up, one may ask an analogue of Question 1 for Griffiths semipositive vector bundles, wondering if, outside the cone spanned by the pushforwards (13), there are other Hermitian or strongly positive Schur forms. If they were all (at least) Hermitian positive one would have an affirmative answer to the original Griffiths' conjecture as stated in [Gri69, p. 247].

However, we point out that these considerations are quite optimistic. For instance, beside the cases listed in Remark 2.4, we do not deduce the Hermitian positivity of $c_{2}(E, h)$ (resp. of $S_{(2,1,0)}(E, h)$ ) by the proof of Theorem 2.2 (resp. of Theorem 0.1 ).

3.3. Other related questions. Let $\mathcal{E}$ be an ample vector bundle over a projective manifold $X$.

As pointed out in Pin18, Xia21, one can study some variants of Griffiths' conjecture. For instance, [Xia21, Conjecture 1.4] asks whether every Schur class $S_{\sigma}(\mathcal{E})$ does admit a positive representative. Such question fits between Fulton-Lazarsfeld theorem and Griffiths' conjecture. Thanks to [Xia21, Theorem A] we know that the answer to this question is affirmative if $|\sigma|=\operatorname{dim} X-1$. Another partial affirmative answer to Xia21, Conjecture 1.4] is given in [DF20, Theorem 4.8], where it is shown that the cohomology classes in the Schur cone coming from push-forwards as in Expression (13) contain a strongly positive form. However, the general problem is open.

Moreover, as observed in [Xia21, Remark 1.5], given a smooth representative $\eta \in S_{\sigma}(\mathcal{E})$ it is not clear how to find a Hermitian metric $h$ on $\mathcal{E}$ such that $\eta=S_{\sigma}(\mathcal{E}, h)$. By virtue of these remarks, one may wonder the following.

Question 2. Given an ample vector bundle $\mathcal{E} \rightarrow X$, is it true that for any partition $\sigma \in \Lambda(k, r)$, there exists a Hermitian metric $h_{\sigma}$ on $\mathcal{E}$ such that $S_{\sigma}\left(\mathcal{E}, h_{\sigma}\right)$ is (Hermitian/strongly) positive?

If $\operatorname{dim} X=2$ and $\mathcal{E}$ is semistable with respect to some polarization, [Pin18, Theorem 1.1] provides an affirmative answer to Question 2, finding a Hermitian metric on $\mathcal{E}$ whose Schur forms are positive. However, it is not clear if such metric is Griffiths positive: see [Pin18, p. 633]. Clearly, an affirmative answer to Question 2 would imply [Xia21, Conjecture 1.4].

We would finally remark that affirmative answers to Xia21, Conjecture 1.4] or Question 2 do not imply Griffiths' conjecture, given that the latter requires the positivity of the Schur forms with respect to one given Griffiths (semi)positive metric on the vector bundle.

\section{REFERENCES}

[BC65] Raoul Bott and S. S. Chern, Hermitian vector bundles and the equidistribution of the zeroes of their holomorphic sections, Acta Math. 114 (1965), 71-112. MR 185607

[BP13] Zbigniew Błocki and Szymon Pliś, Squares of positive $(p, p)$-forms, C. R. Math. Acad. Sci. Paris 351 (2013), no. 1-2, 27-32. MR 3019757 
[Dem12] Jean-Pierre Demailly, Complex Analytic and Differential Geometry, available at https://www-fourier.ujf-grenoble.fr/d̃emailly/manuscripts/agbook.pdf, version of 2012 .

[DF20] Simone Diverio and Filippo Fagioli, Pointwise Universal Gysin formulae and Applications towards Griffiths' conjecture, 2020, arXiv e-prints, arXiv:2009.14587. To appear in Ann. Sc. Norm. Super. Pisa Cl. Sci. (5).

[DP17] Lionel Darondeau and Piotr Pragacz, Universal Gysin formulas for flag bundles, Internat. J. Math. 28 (2017), no. 11, 1750077, 23 pp. MR 3714353

[DPS94] Jean-Pierre Demailly, Thomas Peternell, and Michael Schneider, Compact complex manifolds with numerically effective tangent bundles, J. Algebraic Geom. 3 (1994), no. 2, 295-345. MR 1257325

[Fin20] Siarhei Finski, On characteristic forms of positive vector bundles, mixed discriminants and pushforward identities, arXiv e-prints, arXiv:2009.13107, 2020.

[FL83] William Fulton and Robert Lazarsfeld, Positive polynomials for ample vector bundles, Ann. of Math. (2) 118 (1983), no. 1, 35-60. MR 707160

[Gri69] Phillip A. Griffiths, Hermitian differential geometry, Chern classes, and positive vector bundles, Global Analysis (Papers in Honor of K. Kodaira), Univ. Tokyo Press, Tokyo, 1969, pp. 185-251. MR 0258070

[Gul06] Dincer Guler, Chern forms of positive vector bundles, ProQuest LLC, Ann Arbor, MI, 2006, Thesis (Ph.D.)-The Ohio State University. MR 2709037

[Gul12] - On Segre Forms of Positive Vector Bundles, Canad. Math. Bull. 55 (2012), no. 1, 108-113. MR 2932990

[HK74] Reese Harvey and A. W. Knapp, Positive $(p, p)$ forms, Wirtinger's inequality, and currents, Value distribution theory (Proc. Tulane Univ. Program, Tulane Univ., New Orleans, La., 1972-1973), Part A, 1974, pp. 43-62. MR 0355096

[Li21] Ping Li, Nonnegative Hermitian vector bundles and Chern numbers, Mathematische Annalen 380 (2021), no. 1-2, 21-41.

[LZ20] Ping Li and Fangyang Zheng, Chern Class Inequalities on Polarized Manifolds and Nef Vector Bundles, International Mathematics Research Notices (2020), rnaa317.

[Pin18] Vamsi Pritham Pingali, Representability of Chern-Weil forms, Math. Z. 288 (2018), no. 1-2, 629-641. MR 3774428

[Xia21] Jian Xiao, On the positivity of high-degree Schur classes of an ample vector bundle, Science China Mathematics (2021), https://doi.org/10.1007/s11425-0201868-7.

[Zhe00] Fangyang Zheng, Complex Differential Geometry, AMS/IP Studies in Advanced Mathematics, vol. 18, American Mathematical Society, Providence, RI; International Press, Boston, MA, 2000. MR 1777835

Filippo Fagioli, Dipartimento di Matematica "Guido Castelnuovo", Sapienza Università di Roma, Piazzale Aldo Moro 5, I-00185 Roma.

Email address: fagioli@mat.uniroma1.it 